\title{
Macro- and Micromechanical Behavior of 316LN Lattice Structures Manufactured by Electron Beam Melting
}

\author{
Stefan Roos (D), Carlos Botero, Jonas Danvind, Andrei Koptioug, and Lars-Erik Rännar
}

\author{
(Submitted March 15, 2019; in revised form October 25, 2019; published online November 27, 2019)
}

\begin{abstract}
This work focuses on the possibility of processing stainless steel $316 \mathrm{LN}$ powder into lightweight structures using electron beam melting and investigates mechanical and microstructural properties in the material of processed components. Lattice structures conforming to ISO13314:2011 were manufactured using varying process parameters. Microstructure was examined using a scanning electron microscope. Compression testing was used to understand the effect of process parameters on the lattice mechanical properties, and nanoindentation was used to determine the material hardness. Lattices manufactured from $316 \mathrm{~L}$ using EBM show smooth compression characteristics without collapsing layers and shear planes. The material has uniform hardness in strut shear planes, a microstructure resembling that of solid 316LN material but with significantly finer grain size, although slightly coarser sub-grain size. Grains appear to be growing along the lattice struts (e.g., along the heat transfer direction) and not in the build direction. Energydispersive $x$-ray spectroscopy analysis reveals boundary precipitates with increased levels of chromium, molybdenum and silicon. Studies clearly show that the $316 \mathrm{LN}$ grains in the material microstructure are elongated along the dominating heat transfer paths, which may or may not coincide with the build direction. Lattices made from a relatively ductile material, like $316 \mathrm{LN}$, are much less susceptible to catastrophic collapse and show an extended range of elastic and plastic deformation. Tests indicate that EBM process for $316 \mathrm{LN}$ is stable allowing for both solid and lightweight (lattice) structures.
\end{abstract}

Keywords 316L, additive manufacturing, electron beam melting, ISO 13314:2011, lattice, nanoindentation

\section{Introduction}

Electron beam melting (EBM, sometimes referred to as selective electron beam melting) is a representative of the powder bed beam-based additive manufacturing technologies (Ref 1). So far, the only industrial EBM machine is manufactured by Arcam AB, Sweden. This technology utilizes an electron beam to preheat and melt layers of conducting powders. It is capable of manufacturing components with solid and lightweight parts including near net shape geometries. When comparing the EBM to other PBF technologies, for instance laser-based ones, one should stress few essential differences. EBM process is performed in high vacuum ( $\sim 10^{-5}$ bar) with powder layer preheated prior to melting and with the build area kept at elevated temperatures (commonly about half of the melting temperature of the chosen material). And despite certain advantages over laser-based PBF processes, there is one quite serious issue: EBM has considerably a lower number of industrially available materials (Ref 2). Major groups of parameters related to the most essential process stages are aggregated into two sets, namely the preheating theme and melting theme. The machine

Stefan Roos, Carlos Botero, Jonas Danvind, Andrei Koptioug, and Lars-Erik Rännar, Department of Quality Technology and Mechanical Engineering, Sports Tech Research Centre, Mid Sweden University, Akademigatan 1, 83125 Östersund, Sweden. Contact e-mail: stefan.roos@miun.se. parameters used for melting the material are divided into two subcategories: "contours" and "hatch." Contours are used for the processing of the part surfaces and hatch for the internal bulk material (Ref 3 ).

Flexibility of the process parameter control in EBM allows for quite different strategies of both contour melting and hatching, including the number of contours, spacing between each contour line and contour overlap area with hatch, beam motion pattern during hatching and its parameters, beam parameters during contouring and hatching stages, etc. This general pattern with both contour melting and hatching is common for solid parts with rather large cross-sectional area. In the cases when component cross-sectional area becomes smaller and smaller, the pattern can be changing. Figure 1 exemplifies the change in the melting pattern of an oval crosssectional area with consecutively decreasing dimensions. In this particular example, initially three contour lines with the hatching inside them are presented in Fig. 1(a) and (b). At some point, the space is only enough for the three contours (Fig. 1c). Further reduction in cross section will leave only two contour lines and then just one oval contour (Fig. 1d and e). Further reduction in the melt area leads to the activation of the built-in "block offset" function reducing the beam path to a single line along the median of the cross-sectional area, or even a single beam spot (Fig. 1f) (Ref 4).

Lightweight structures commonly have very small crosssectional area dimensions. Two typical examples of such structures are represented by regular lattices (also referred to as net, mesh or cellular structures) with repetitive cells of defined geometry and by the stochastic foams or reticulated mesh structures (Ref 5). One of the major parameters of such structures defining their properties and application areas is the porosity commonly defined as a ratio of the metal and air volumes in the component outer envelope (Ref 5, 6). Element 


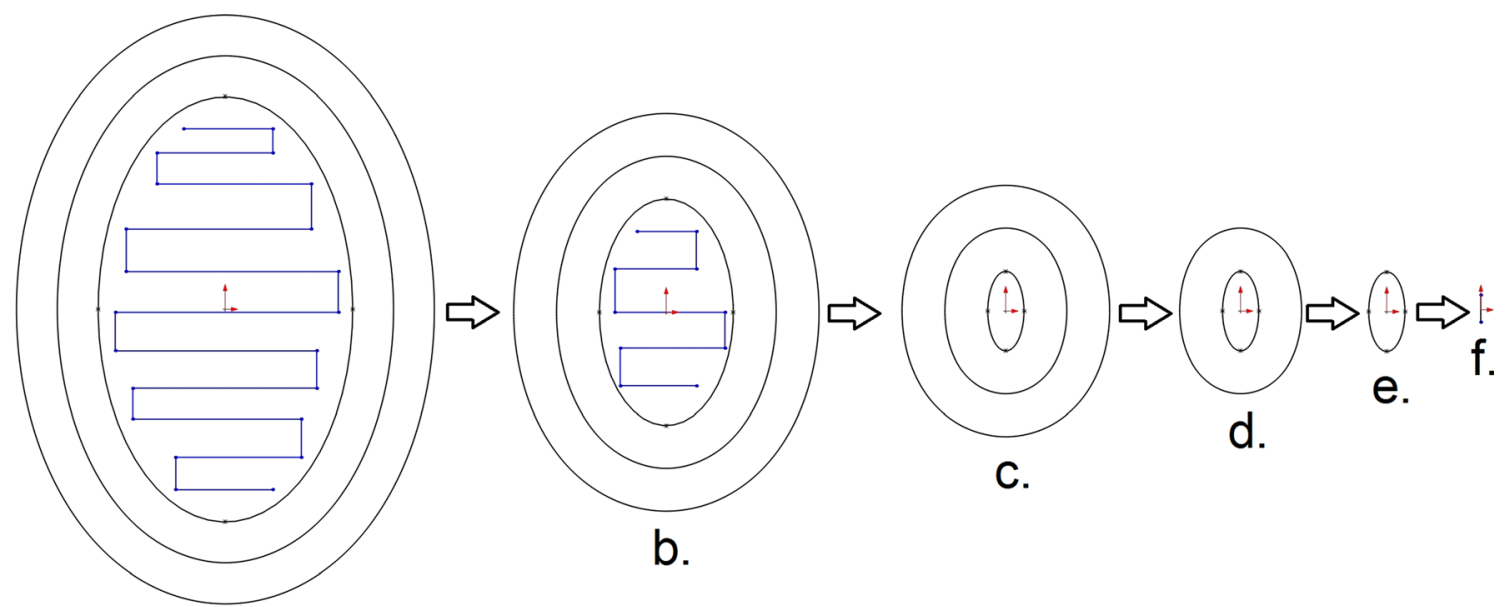

a.

Fig. 1 Electron beam scanning pattern changes when the cross section of the melt area decreases in size (left to right). Contour melting in black and hatch pattern in blue (Color figure online)

cells of regular lattices commonly consist of beams (struts) connected together. Lightweight regular lattices commonly have quite thin beams, and in such cases melting strategy changes from trace melting (as shown in Fig. 1f) to a spot melting. This type of melting defines the differences in material properties and specific rough surfaces of the lattice elements. The properties of lattice structures manufactured in Ti6Al4V using EBM (Ref 5, 7-9) and laser-based PBF systems (Ref 1012) have been explored in several studies. But lightweight lattice structures made from Ti6Al4V are commonly having undesirable fracture modes sometimes leading to a catastrophic collapse of the entire structure, which is partially due to the brittleness of the material. Thus, there is a significant interest to the properties of the lattice structures made from a ductile material, for example austenitic stainless steel 316L. This material is available for industrial manufacturing in several laser-based PBF machines. And in several studies properties of both solid (Ref 13) and lattice structures (Ref 14, 15) were studied. And though stainless steel $316 \mathrm{~L} / 316 \mathrm{LN}$ is currently not a commercially supported material by Arcam AB, solid samples have been successfully manufactured and solid material properties were analyzed (Ref 16, 17).

One of the main problems of studying lightweight lattice structures is studying the properties of material in their elements. In the case of lattices, their beams can have very small cross-sectional area dimensions, often comparable to the dimensions of Rockwell or Brinell testing probes (ASTM E10, ASTM E18). It is also impossible to expect any reasonable results by applying macroscopic hardness probes to the elements of flexible lattices without lattice encapsulation. Recently, the method of nanoindentation gains popularity for the small-scale studies of mechanical properties in small volumes of material (Ref 18, 19). This method allows measuring stress-strain curves, Young's modulus and hardness at micro-/nanoscale. The results of nanoindentation will commonly not coincide exactly with the values acquired using Rockwell or Brinell methods. (Their probes are large enough to get certain averaging of the properties from nanoscale size structures.) But nanoindentation is capable of predicting hardness tendencies while yielding much more detailed hardness breakdown to nanoscale. Also, due to the fact that the actual loads applied are small, the spring-like action of the lattice elements has a minor impact of the apparent response. Thus, nano- and microscale mechanical testing methods like nanoindentation could be quite suitable for studying the mechanical properties of material in the elements of lightweight structures including lattices.

The aim of present study was to manufacture different lattice structures in $316 \mathrm{LN}$ stainless steel using an Arcam EBM machine and to investigate both macro- and micromechanical properties of the samples manufactured, using unaltered CAD models but with different process parameter settings, using compression testing and nanoindentation.

\section{Method}

\subsection{CAD Model Design}

The lattice structure CAD model was designed using a combination of two programs, namely SolidWorks 2016 (Dassault Systems, France) and Magics 20.04 (Materialise, Belgium). A single $3 \times 3 \times 3 \mathrm{~mm}$ octagon-type unit cell with $0.2 \mathrm{~mm}$ in diameter beams was modeled in SolidWorks (Fig. 2a). The single cell CAD file was imported into Magics and checked for errors, and an array of $10 \times 10 \times 15$ cells $(X$, $Y, Z$ ) was prepared. Corresponding outline of the sample is chosen to satisfy the dimensions for the compression testing under the ISO13314-2011 (Ref 20) standard. The outline of the final lattice structure has the dimensions of $30 \times 30 \times 45(X$, $Y$, Z) $\mathrm{mm}$.

To calculate the porosity, the overall volume of a single unit cell and the volume of the struts were calculated from the designed model CAD file. The calculated theoretical porosity of the octagonal unit cell is $97.7 \%$.

\subsection{Fabrication}

Manufacturing of the samples, lattice and solid, was carried out in-house at the Sport Tech Research Centre at Mid Sweden University in Östersund, Sweden. Solid samples used for reference in comparison with lattice samples were manufac- 

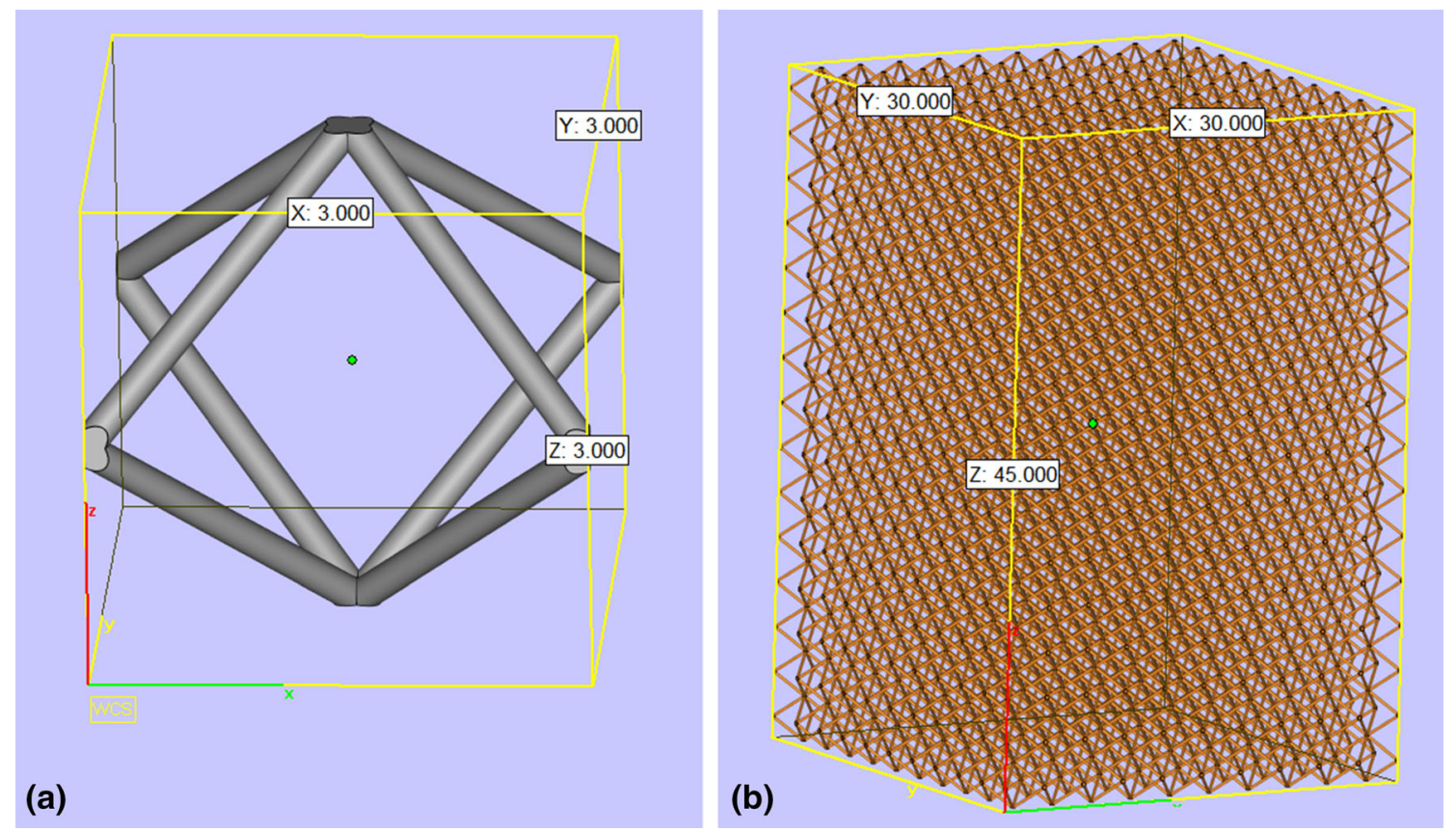

Fig. 2 Graphical representations of a single $3 \times 3 \times 3 \mathrm{~mm}$ octagon unit cell (a) and a $10 \times 10 \times 15$ cell lattice structure (b) consisting of multiplied $3 \times 3 \times 3 \mathrm{~mm}$ octagon unit cells. Figures are prepared using Magics software

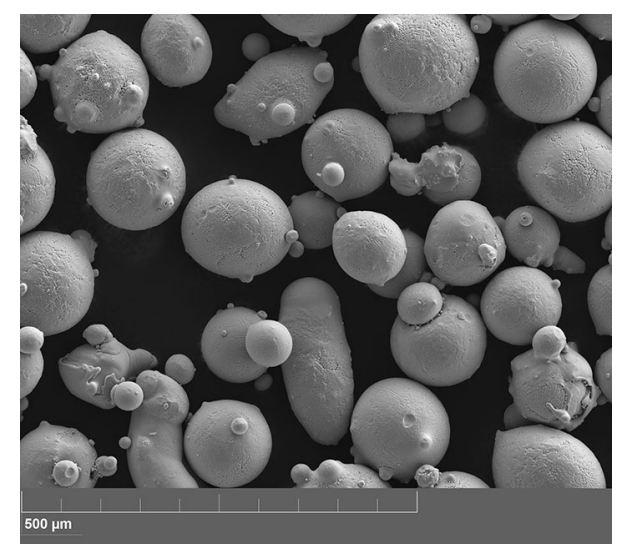

Fig. 3 SEM image of $316 \mathrm{LN}$ powder used for lattice manufacturing

tured using already developed themes for previously performed studies (Ref 16, 17).

2.2.1 Powder. Used 316LN powder was acquired from Carpenter Powder Products AB (Torshälla, Sweden) and has a 53-150 $\mu \mathrm{m}$ grain size range with quite round particles having relatively few satellites (Fig. 3); though some oddly shaped powder grains are present, it did not influence the overall powder flowability. The powder had been used in the EBM process and recycled in standard Arcam powder recovery system several times before the fabrication of the lattice samples.

2.2.2 Lattice Samples. The Arcam EBM process utilizes different groups of settings, called themes, defined for each used material. As stainless steel $316 \mathrm{LN}$ is not supported by the embedded themes with Arcam EBM machines, themes used for fabricating the lattices were determined experimentally. A number of small test samples of lattice structures were manufactured using an ARCAM A2 machine with varying parameter settings. Finally, a theme providing lattice structures with best quality of small features was selected. Two more themes were generated with the beam power some higher and some lower than in the "best available choice" one. These themes will be referred to as "Light," "Medium" (best-quality parameter set) and "Heavy" referring to the expected final density of the lattice due to the thinner or thicker expected beam dimension. Corresponding beam current setting was $3 \mathrm{~mA}$ for Light, 5, $6 \mathrm{~mA}$ for Medium and $7 \mathrm{~mA}$ for the Heavy sets. The three different themes were used to manufacture eight samples of lattice structures each, resulting in a total of 24 lattice samples of ISO 13314-2011 compliant dimensions (Fig. 4). It is known that the first few millimeters of the build (near the start plate) may differ from the rest, as it takes some time to stabilize the thermal conditions in and around the build. The lattices therefore were placed $3 \mathrm{~mm}$ above the start plate. Support structures were not used, and in the final build design lattices were "free floating" without any support structures. Same CAD geometry was used for all the samples forming three sets of eight lattices each separated by 5 -mm-thick solid metal walls (Fig. 4) manufactured with the parameters optimized for the solid 316LN components. The addition of solid components around the lattice ones helps to maintain consistent stable temperature in all consecutive build layers. In Fig. 4, the sets of samples manufactured with three different parameter settings are color coded in gray, green and red, and the solid walls are depicted in orange.

2.2.3 Post-processing. In EBM process, the powder around all components is semi-sintered and needs to be removed before component mechanical testing. This was carried out using a standard powder recovery system (PRS) provided by Arcam AB. The PRS uses working powder in a flow of compressed air to blast the sintered material away and 
recover the powder material for further use. Blasting time was approximately 3-10 min per lattice sample depending on the porosity (lighter sample allowed for shorter blasting time). No further post-processing was carried out.

\subsection{Quasi-Static Compression Testing}

Mechanical properties of the lattices were tested following the ISO 13314-2011 standard (Ref 20). The lattices were tested "as built" without any additional post-processing except the powder removal. Lattices used for compression testing were weighed and measured using a VI-1-mg resolution scale (Acculab, USA) and a generic caliper with $0.05 \mathrm{~mm}$ resolution. Sample dimensions and weight are presented in Table 1. The compression tests were carried out at room temperature $\left(20{ }^{\circ} \mathrm{C}\right)$ using an Instron 5969 Universal testing system (Instron, USA) equipped with a 50-kN load cell using "Bluehill 3" software. ISO $13314-2011$ specifies a strain rate between $10^{-3} / \mathrm{s}$ and $10^{-2} / \mathrm{s}$. A strain rate of $0.005 / \mathrm{s}$ was chosen, and when applied to the sample geometry of $45 \mathrm{~mm}$ height, it results in an anvil motion speed of $13.5 \mathrm{~mm} / \mathrm{min}$.

2.3.1 Data Processing. From the compression test, raw data energy absorption $(W)$ was calculated using $W=$ $\frac{1}{100} \int_{0}^{\varepsilon_{\text {ple }}} \sigma \mathrm{d} \varepsilon$ where $\sigma$ is the compressive stress and $\varepsilon_{\text {ple }}$ is the

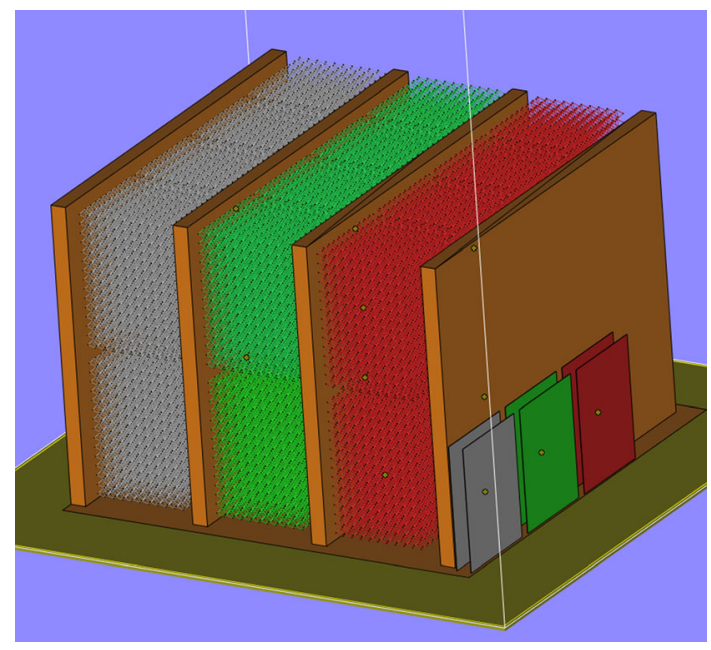

Fig. 4 Placement of the lattice samples separated by the solid walls in the CAD file of the build generated by Magics software. Color coding for the different parameter settings used for lattices and the walls is gray, green and red corresponding to "Light," "Medium" and "Heavy" parameter sets; orange corresponds to the set used for solid samples (Color figure online) plateau end strain (Fig. 5). The energy absorption efficiency $\left(W_{\mathrm{e}}\right)$ was calculated using $W_{\mathrm{e}}=\frac{W}{\sigma_{130} * \varepsilon_{\mathrm{ple}}} \times 10^{4} . \sigma_{\mathrm{pl}}$ was calculated based on the experimental stress-strain curves, and $\sigma_{130}$ was determined using $\sigma_{130}=\sigma_{\mathrm{pl}} * 1.3$.

Equations and nomenclature are used according to ISO13314:2011 standard (Ref 20).

\subsection{Microstructure and Chemical Composition Analysis}

The grain dimensions were examined using "particle analysis" in software "ImageJ" (National Institutes of Health, USA). Cross sections of lattices manufactured with all three chosen themes were analyzed. In each analyzed strut, a bounding box was defined for grain size quantification. The box was defined to include the largest possible homogeneous mass of grains while excluding small grained clusters at the periphery of the cross section under analysis. Grain size of solid $316 \mathrm{LN}$ sample was also analyzed in the same way for comparison. The elemental composition of the material in the cross-sectional cuts and grain boundary inclusions were determined using a Tescan Maia 3 SEM (Tescan, Brno, Czech Republic) setup for energy-dispersive x-ray spectroscopy (EDS). The Maia 3 SEM was also used for producing images of the $316 \mathrm{LN}$ powder used to build the lattices as well as images of the cross sections revealing the grain boundaries used for grain size analysis.

\subsection{Sample Preparation}

Microstructure analysis and nanoindentation test lattices were first protected by encapsulation in acrylic resin. Specimens were then ground from the top face (reducing the height of the samples in build direction Z) using a 240-grit sandpaper to obtain $x y$ cross sections in desired lattice positions. Corresponding samples revealing desired lattice cross sections were further prepared by careful manual polishing using diamond abrasive pastes with grains down to $1 \mu \mathrm{m}$. After that, the final polishing was performed using colloidal silica suspension. Polished specimens were chemically etched using "Kroll" reagent. The series of etching stages at increasing times in the range of 15-40 min with consecutive microscopy observation was performed to avoid excessive etching but allowing revealing different microstructural features. Corresponding samples for the microstructure analysis were prepared with the cross sections normal to the build direction.

\subsection{Compression Nanoindentation Tests}

In order to evaluate the intrinsic mechanical behavior of the $316 \mathrm{LN}$ material in the lattices, nanoindentation tests were locally performed on the polished cross sections of the struts. Accounting for the thickness of the struts, and taking into consideration the microstructural features of the material, a

Table 1 Parameters of the lattice samples used for compression testing

\begin{tabular}{|c|c|c|c|c|c|}
\hline \multicolumn{4}{|c|}{ Average dimensions, mm (SD) } & \multirow[b]{2}{*}{ Average weight, g (SD) } & \multirow[b]{2}{*}{ Average porosity, \% (SD) } \\
\hline Sample & Length & Width & Height & & \\
\hline Light & $29.75(0.058)$ & $29.75(0.058)$ & $44.625(0.126)$ & $18.43(0.244)$ & $94.2(0.08)$ \\
\hline Medium & $29.925(0.05)$ & $29.875(0.05)$ & $44.875(0.05)$ & $29.72(0.265)$ & $90.7(0.06)$ \\
\hline Heavy & $30(0)$ & $30(0)$ & $44.875(0.05)$ & $37.73(0.226)$ & $88.3(0.06)$ \\
\hline
\end{tabular}




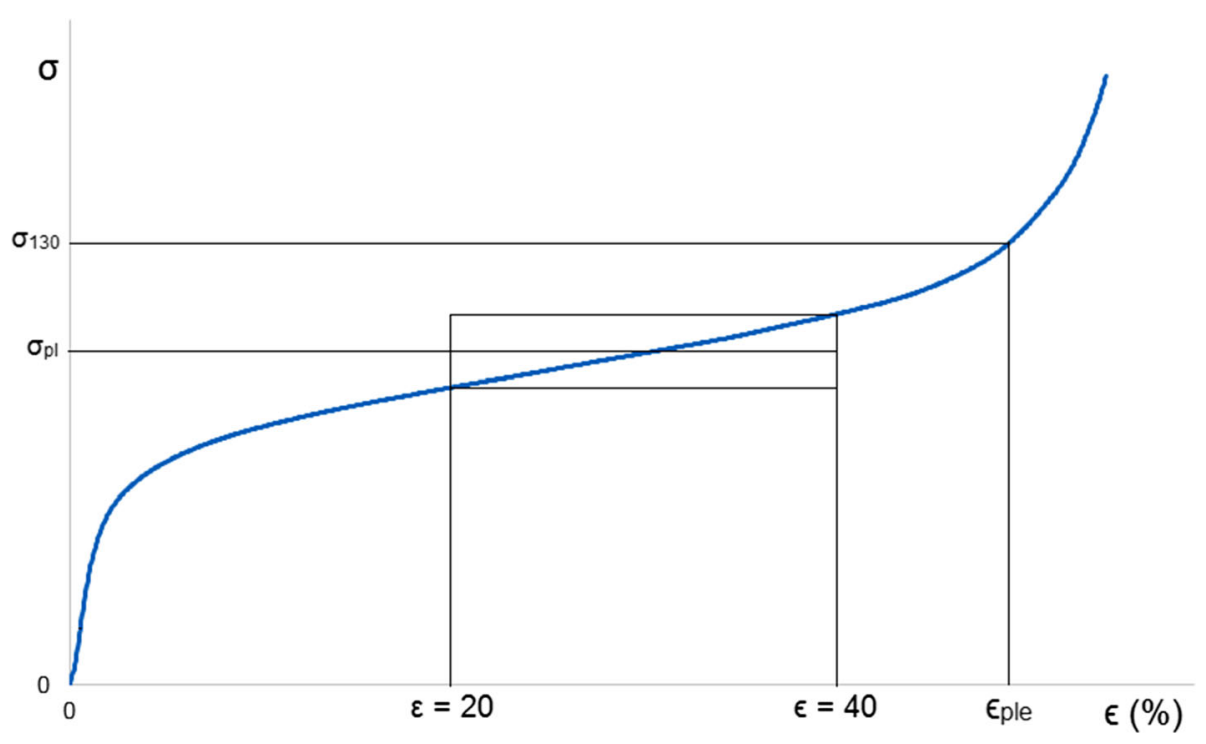

Fig. 5 Generic stress/strain plot describing values used for calculations

Table 2 Calculation results for energy absorption $(W)$ and energy absorption efficiency ( $\left.W_{\mathrm{e}}\right)$ for EBM built lattice structures in $316 \mathrm{LN}$ stainless steel

\begin{tabular}{|c|c|c|c|c|c|c|}
\hline \multirow[b]{2}{*}{ Sample } & \multicolumn{2}{|c|}{ Light } & \multicolumn{2}{|c|}{ Medium } & \multicolumn{2}{|c|}{ Heavy } \\
\hline & $W, \mathbf{k J} / \mathbf{m}^{3}$ & $W_{\mathrm{e}}, \%$ & $W, \mathbf{k J} / \mathbf{m}^{3}$ & $W_{\mathrm{e}}, \%$ & $W, \mathbf{k J} / \mathbf{m}^{3}$ & $W_{\mathrm{e}}, \%$ \\
\hline 1 & 2.10 & 69.93 & 8.98 & 71.85 & 15.82 & 71.36 \\
\hline 2 & 2.32 & 70.12 & 10.23 & 72.50 & 17.13 & 71.94 \\
\hline 3 & 2.32 & 70.28 & 10.73 & 73.03 & 17.50 & 71.97 \\
\hline 4 & 2.31 & 70.87 & 9.62 & 72.51 & 17.55 & 72.09 \\
\hline Average (SD) & $2.26(0.11)$ & $70.30(0.40)$ & $9.98(0.76)$ & $72.47(0.49)$ & $17.00(0.81)$ & $71.84(0.33)$ \\
\hline
\end{tabular}

maximum penetration depth of $h_{\max }=500 \mathrm{~nm}$ was chosen. Indentation profiles were acquired across the cross sections of the struts. Matrices of minimum three indentation columns and a variable number of rows depending of the thickness of the struts were performed, first indentations starting from the inner parts and subsequently moving to the periphery of the tested cross section. Separation between individual indentation positions was $12.5 \mu \mathrm{m}$. Residual imprints were investigated using optical microscopy (OM) and scanning electron microscopy (SEM). Nanoindentation tests were carried out in a MTS Nanoindenter XP at a constant strain rate of $0.05 / \mathrm{s}$, using a Berkovich indenter with its area function calibrated using a fused silica standard. The continuous stiffness measurement (CSM) module was activated to continuously record the load $(P)$, penetration $(h)$ and the contact stiffness $(S)$ data. Hardness $(H)$ and elastic modulus $(E)$ were evaluated as a function of the penetration depth based on the model proposed by Oliver and Pharr (Ref 21).

\section{Results and Discussion}

\subsection{Lattice Structures and Macromechanical Behavior}

The lattice structures were measured for size and weighed before compression testing. Corresponding lattice porosity (defined here as the volumetric air-to-material ratio of the entire lattice) was determined basing on the dimensions and weight of the lattice samples (see Table 1).

The results of the energy absorption calculations based on the test sample compression tests show that the heavier lattices can absorb more energy when compressed while the efficiency of energy absorption is similar between all lattices of tested densities. Cumulative data from the compression tests of the lattice samples are presented in Table 2 .

The results from the compression testing show that Heavy (most dense) lattices have the highest compression strength, followed by the Medium and Light ones. Figure 6 presents representative stress-strain curves for the lattices of different densities. It is clear that lattices made using the same CAD file have similar functional compression behavior, showing extended plasticity with increasing strength connected to increasing structure density. Thus, it is possible to control the lattice structure compression strength by only changing the process parameter settings within certain range without changing the design geometry.

It should be noted that compression behavior and collapse of the lattice structures made from 316LN stainless steel significantly differ from the one reported for the Ti64 lattice structures commonly showing clear shear planes (Ref 5, 8-10). $316 \mathrm{LN}$ lattices show bulging and rather smooth compression damage without tendencies to catastrophic collapse (Fig. 7). 
Most probably, it is due to the properties of $316 \mathrm{LN}$ steel having significantly higher ductility than Ti6Al4V, and thus, lattice elements are less prone to brittle fracture. Most likely, the resulting behavior with the initial elastic compression and extended plastic deformation of the lattice almost until it is turned to a solid piece is a combination of material properties and unit cell geometry. But no definite conclusion can be drawn as to which factor is the dominant without additional extensive testing.

\subsection{Microstructural Analysis and Chemical Composition}

$316 \mathrm{LN}$ grain size in the lattice struts is finer than what have been previously reported for the solid EBM-made material, see Table 4. One can speculate that this is a result of faster cooling due to a smaller amount of melted material per manufacturing layer. From the visual analysis of different strut cross sections, it can be concluded the Light-density lattices (smallest strut cross-sectional area) have the finest grain structure and the high-density lattices the coarsest. This is, however, not supported by the grain size measurements. There are two possible reasons for such discrepancy. Firstly, the eye can react to the linear dimensions of the grains (closer to "width" and "length"), while the grain size measurements refer to the surface areas. Also, as the dimensions of the grains are different

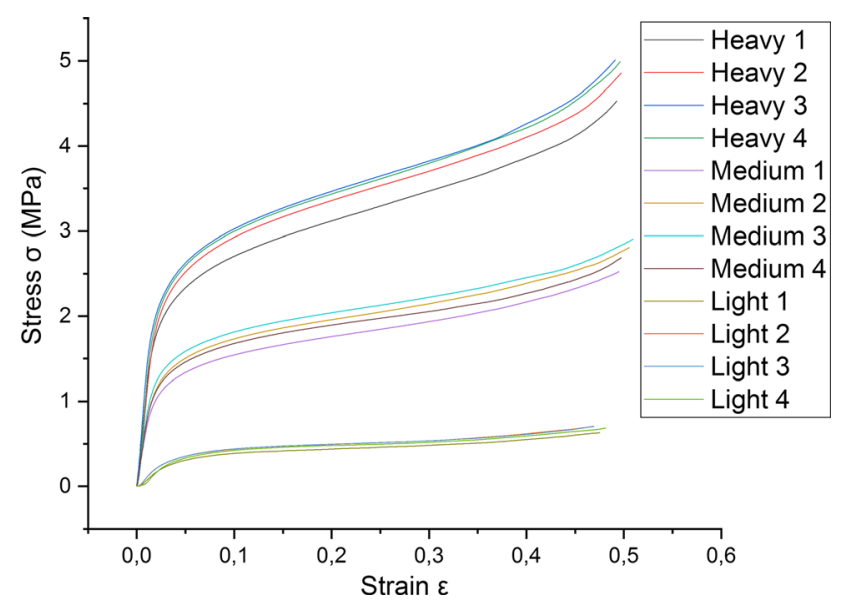

Fig. 6 Stress vs. strain plot of lattice structure compression testing. Lattices with the same EBM process theme are neatly grouped together for different parts of the strut cross section (Fig. 8, Table 4 last two columns), the result of grain area measurements may be biased by the choice of the cross-sectional area analyzed. It was also noticed that average grain size in the cross sections of the lattice nodes (where few struts meet) is larger than in the cross section at the middle of the struts. Thus, all the measurements of the grain dimensions were performed on the strut cross sections equidistant from the adjacent nodes. Figure 8 presents typical image for the polished and etched lattice sample normal to the build direction. Analysis of the sample reveals two distinctive grain dimensions with coarser grains in the center of the cross section (Fig. 8a) and finer grains in clusters along the periphery of the sample (Fig. 8b).

Grain areas measured using same protocol in the lattice strut cross sections (normal to the build direction) are at least 10 times smaller than for the solid $316 \mathrm{LN}$ samples (cross sections normal to the build direction) made from the same powder batch in the same EBM machine. Grain dimensions measured for the solid $316 \mathrm{LN}$ samples made by EBM in present work are similar to what can be estimated using images presented in (Ref 22). Large standard deviation values of the measured grain surface area values (Table 4) are coming from the wide distribution of the grain sizes. The EDS material composition analysis shows that the chemical composition, after EBM processing, for 316LN (ASTM A240) is met for the scanned area, except for a slightly to high level $(0.05 \%)$ chromium (see Table 3). Figure 9 presents the choice of EDS scanning area. The scanning area was chosen to avoid grain boundary precipitates, including high concentrations of certain elements, to impact the results.

From the EDS line scan (Fig. 10), it can be seen that the precipitates have higher levels of chromium, molybdenum and silicon while the levels of nickel and iron are lower. These results are similar to the solid EBM-manufactured 316LN from the study performed by Zhong et al. (Ref 16), but the increased levels of silicon in precipitates were not mentioned in that study.

Comparative analysis of the material composition for the precursor powder, lattice and solid $316 \mathrm{LN}$ samples is given in Table 3.

The 316LN lattice microstructure grain size is finer than what have been previously reported for solid EBM-manufactured 316LN samples, see Table 4. The measurement of grain size in solid material is based upon an in-house-manufactured

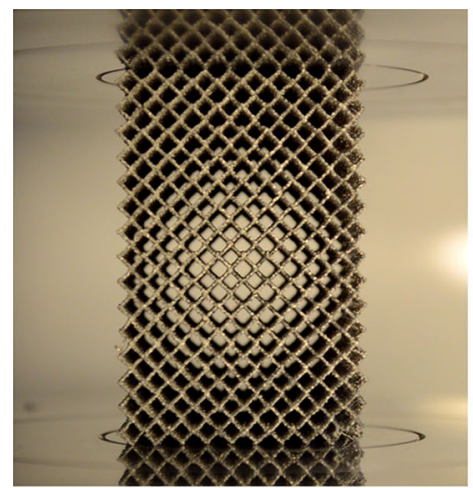

(a) $\varepsilon=0$

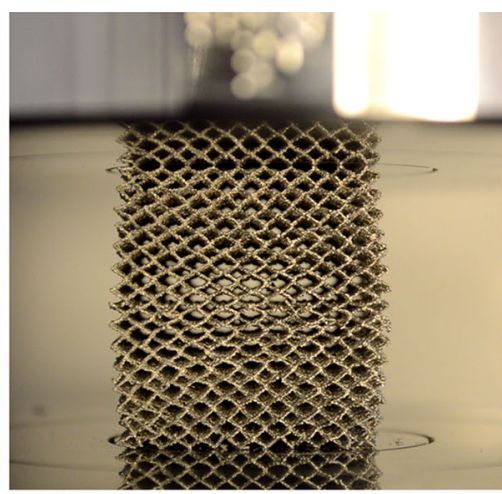

(b) $\varepsilon=0,25$

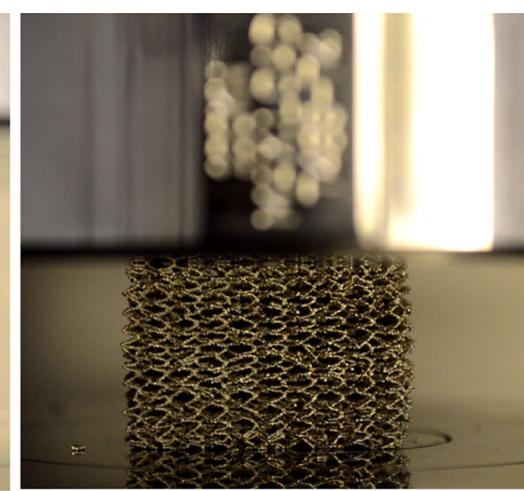

(c) $\varepsilon=0,5$

Fig. 7 Lattice structure at different stages of compression 
sample and from Fig. 5(b) in (Ref 22) both showing similar grain sizes.

There is a clear difference in the grain morphology between the bulk and lattice samples manufactured via EBM. It is commonly attributed to the difference in the thermal conditions in two cases. The correlation between cooling rate and average grain dimensions is well supported by research (Ref 23, 24). Also supported is the correlation between cooling rate and dendrite arm spacing (Ref 25). The equation $\lambda_{1}=80 \dot{\mathrm{T}}^{-0,33}$, where $\lambda_{1}$ is the dendrite arm spacing and $\dot{\mathrm{T}}$ is the cooling rate, has been used previously to estimate L-PBF cooling rates, yielding the values of $5.8 \times 10^{5} \mathrm{~K} / \mathrm{s}(\operatorname{Ref} 26),>10^{6} \mathrm{~K} / \mathrm{s}$ (Ref 27) and $4.8 \times 10^{6}-2.6 \times 10^{5} \mathrm{~K} / \mathrm{s}$ (Ref 24) for bulk material samples. An estimated average dendrite spacing of the EBM-processed lattice samples of $2.7 \mu \mathrm{m}$ found in this study suggests a cooling rate of $2.6 \times 10^{4} \mathrm{~K} / \mathrm{s}$. In the previously mentioned studies, the laser power was varied between 200 and $2000 \mathrm{~W}$ (Ref 24), 110 and $200 \mathrm{~W}$ (Ref 27) and fixed at $380 \mathrm{~W}$ (Ref 26). In the present study, lattices were manufactured with the beam power varied in the range 180$420 \mathrm{~W}$, but the cooling rate estimated using the abovementioned expression is an order of magnitude lower. For possible explanation, one should consider the difference in the thermal history of the large solid and lattice samples. Firstly, EBM processing happens at elevated temperatures, with the target value in this study of $830{ }^{\circ} \mathrm{C}$ for $316 \mathrm{LN}$, with continuous heat waves propagating from the sample surface toward the

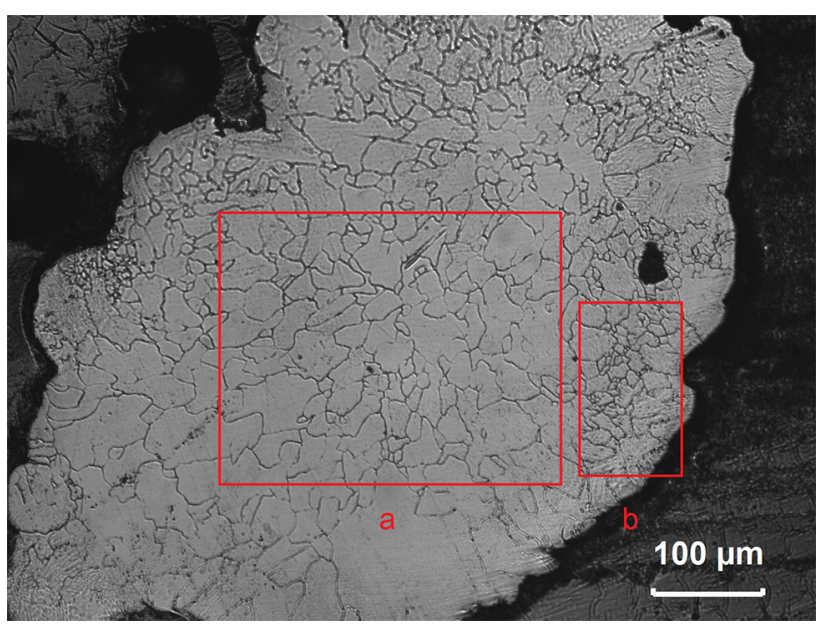

Fig. 8 Typical lattice strut cross section normal to the build direction; boxes (a) and (b) highlight differences in grain size depending on location in the strut cross section. Average grain area for the inner parts of the cross section is $435 \mu \mathrm{m}^{2}$ (a) and $95 \mu \mathrm{m}^{2}$ for the periphery (b) start plate during each melt sequence. During EBM processing, all components are surrounded by the mass of semi-sintered powder, which has certain heat insulation properties. Small cross section of the lattice struts has lower capacity for the heat transfer as compared to the bulk, solid samples having significant cross section. Thus, smaller capacity for the heat transfer from the top layers should result in lower solidification rates. Despite the lower solidification rate leading to coarser sub-grains, the grains are finer. An explanation for this could be that the lattice only sees less than $3 \%$ of the energy input compared to a solid sample of the same dimensions. Considering the high surface area-to-mass ratio of the struts, lower energy input and reduced heat transfer capability of thin lattice struts, one could hypothesize that the average temperature in the solid material therefore is higher, causing an annealing effect of already solidified material, which facilitates grain growth. In addition, in the used ARCAM machine the temperature of the whole build is slowly decreasing for some hours after completion without active cooling. It contributes to a complex thermal history of the material and affects the final grain shape and dimensions, and inter-grain precipitation. Due to the lack of control over the thermal history for the components of different shapes placed in different parts of the processed layer and their final cooling, the task of predicting the final grain morphology and alloy element

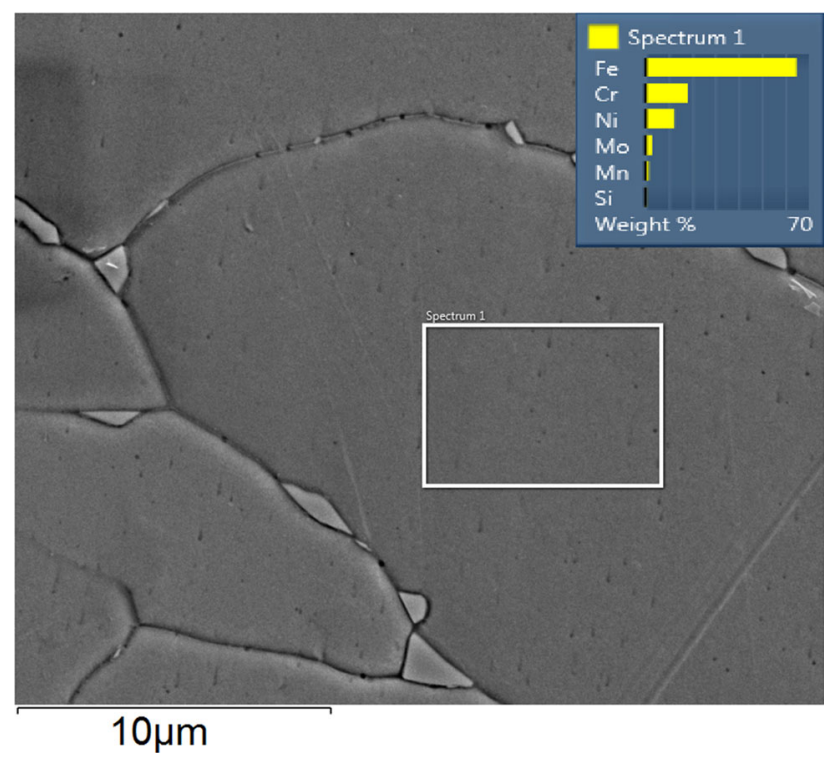

Fig. 9 SEM image of lattice cross-sectional microstructure, white rectangle representing the area scanned using EDS for determining material composition after EBM processing. Material composition spectrum is presented in Table 3

Table 3 Chemical compositions of various $316 \mathrm{LN}$ materials based on the EDS spectrum analysis

\begin{tabular}{|c|c|c|c|c|c|c|}
\hline & Si, wt.\% & Cr, wt.\% & Mn, wt.\% & Ni, wt.\% & Mo, wt.\% & Fe, wt. \% \\
\hline 316LN material spec (ASTM A240) & $0-0.75$ & $16-18$ & $0-2$ & $10-14$ & $2-3$ & Bal. \\
\hline Powder manufacturer spec. & 0.5 & 17.6 & 1.7 & 12.3 & 2.46 & Bal. \\
\hline Lattice samples (EBM) & 0.58 & 18.05 & 1.53 & 12.26 & 2.86 & 64.72 \\
\hline Lattice sample precipitate (EBM) & 1.09 & 29.74 & $\ldots$ & 5.18 & 12.13 & 51.86 \\
\hline Bulk material (EBM) [16] & 0.53 & 17.4 & 1.5 & 12.4 & 2.5 & Bal. \\
\hline
\end{tabular}



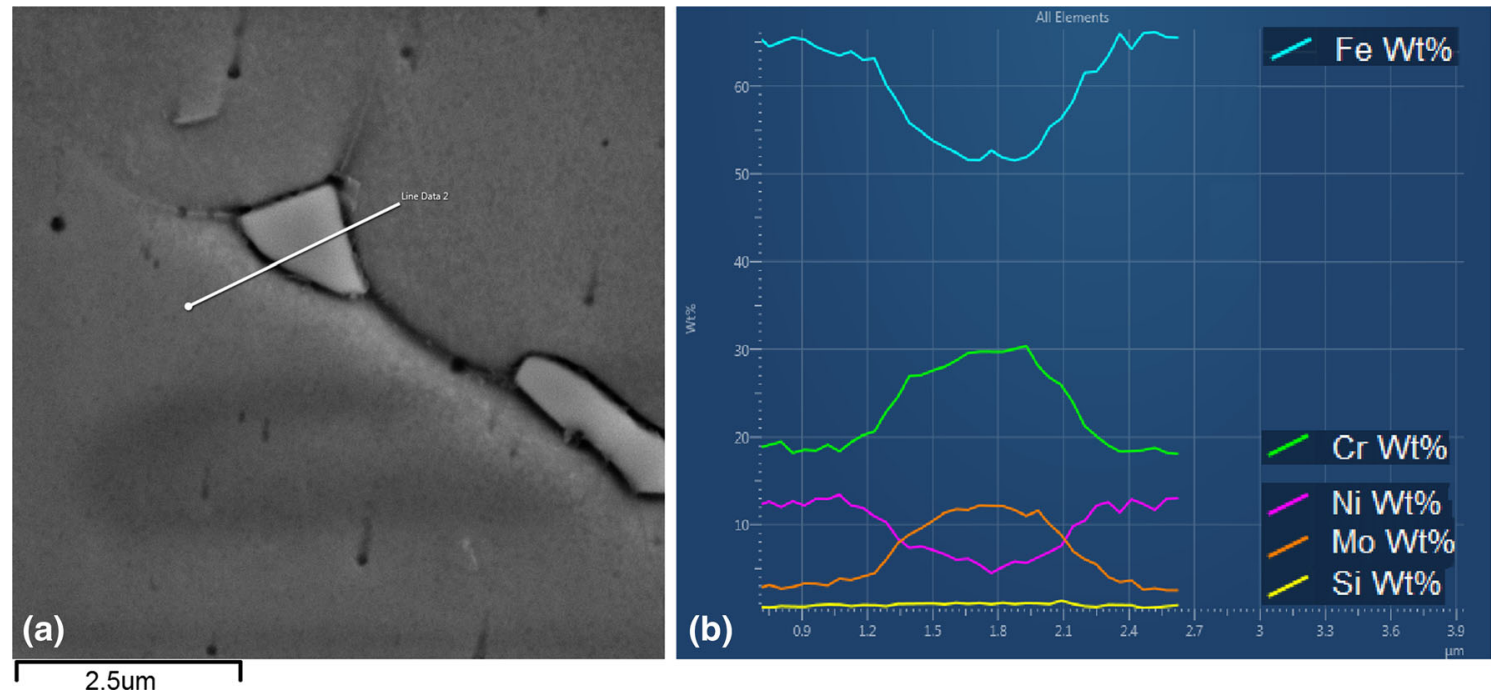

Fig. 10 Line EDS scan of grain boundary precipitate (a) showing increased levels of chromium, molybdenum and silicon (b)

Table 4 Results from grain size measurements: average for whole strut cross section for the lattices made with different parameter settings (Light, Medium and Heavy) and separately in the middle (Box 1) and periphery (Box 2) of the strut for the "Medium" lattice

\begin{tabular}{|c|c|c|c|c|c|c|}
\hline & Light & Medium & Heavy & Solid & Box 1 & Box 2 \\
\hline Mean area, $\mu \mathrm{m}^{2}(\mathrm{SD})$ & $495(860)$ & 337 (467) & $599(960)$ & 4787 (4132) & $436(509)$ & $95(133)$ \\
\hline Number of grains measured & 290 & 204 & 311 & $20[22]$ & 113 & 55 \\
\hline
\end{tabular}

segregation at the grain boundaries becomes quite complex. So far, it is only possible to draw rather general conclusions, as it is carried out in the present study.

It is typically stated that in the powder bed beam-based AM materials the grain elongation follows the build direction (Ref 17, 22, 28). Analysis of the lattice cross sections made along the building direction shows that grains are elongated along the struts (Fig. 11), rather than along the build direction. It has been shown that grains grow along the direction of the thermal gradient (Ref 23). The heat from the electron beam likely flows through the lattice struts due to the insulating properties of the powder, explaining the grain growth deviation from the build direction seen.

\subsection{Intrinsic Micromechanical Behavior of 316LN in Lattices}

An optical image of one of the indented struts in the Lightdensity lattice is presented in Fig. 12 illustrating the changes of the grain and sub-grain boundary exposure with increasing etching time. The matrix of residual nanoindentation imprints can be easily discerned in the polished struts without etching, as evidenced in the optical image in Fig. 12(a). As the indented specimens are etched, the grain boundaries of the material are revealed in the cross section, as it can be seen from the image in Fig. 12(b) in the "etched condition." Further etching of the specimens leads to an "over-etched" condition, in which the underlying sub-grain structure can be intuited; it is more readily visible in some zones of the periphery of the struts (Fig. 12c, 13).

In Fig. 13, a magnified detail of the nanoindentation imprints is presented. The subjacent sub-grain structure is

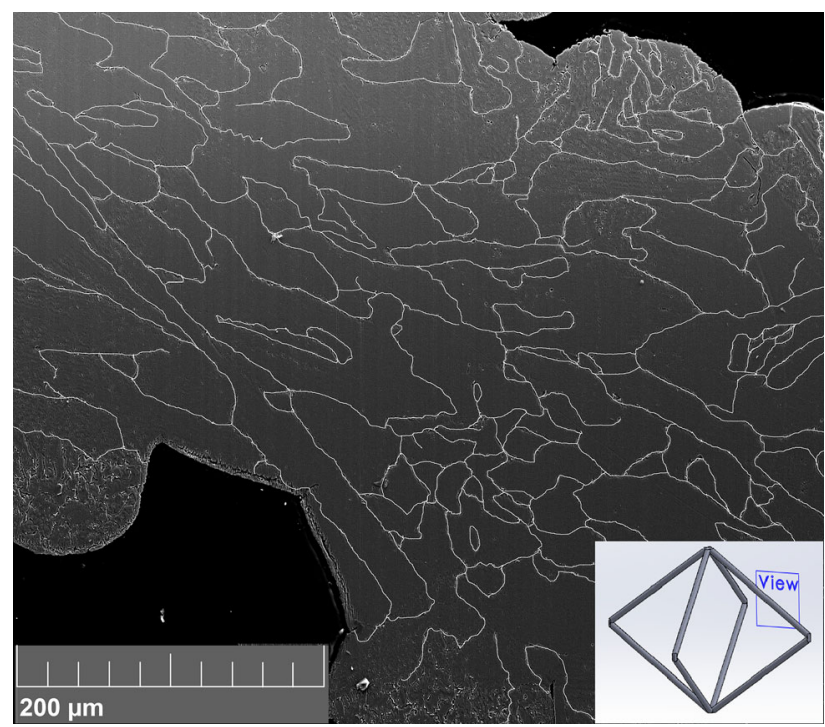

Fig. 11 Typical cross section of the lattice strut with the cut plane along the strut axis (insert showing the cross-sectional area in the lattice cell) illustrating the predominant elongation axis of the grains

evidenced in the periphery of the sample. From this image, it is worth noting that the residual imprints of the nanoindentations are well inscribed within the grains of the material and that they are comparable in size with the characteristic length scale of the sub-grain structure.

Looking more into detail at the sub-grain structure and comparing the lattice sub-grain structure to a solid sample sub- 
grain structure, see Fig. 14, the lattice sub-grain structure is similar to that of a solid sample. The orientation is nonequiaxed, and the sub-grains of the lattice are in the same order

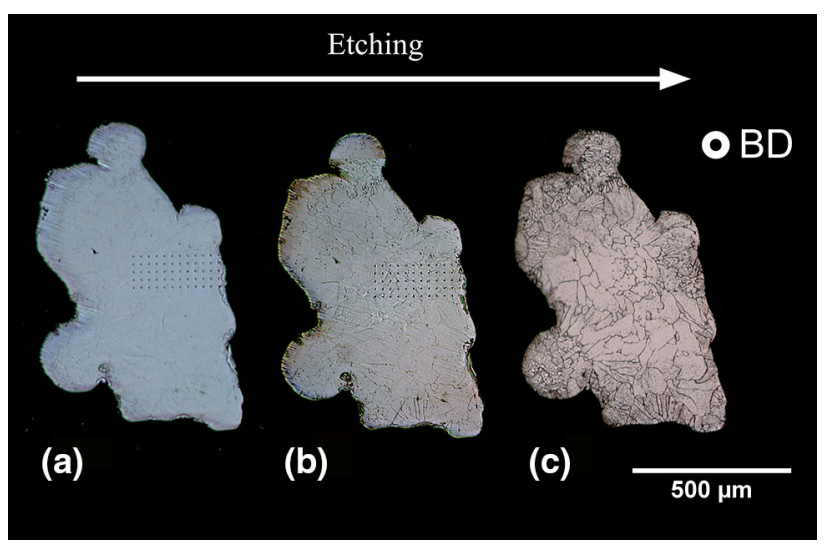

Fig. 12 Indented cross section of one strut in the Light-density lattice. Non-etched condition (a), etched condition (b) and overetched condition (c)

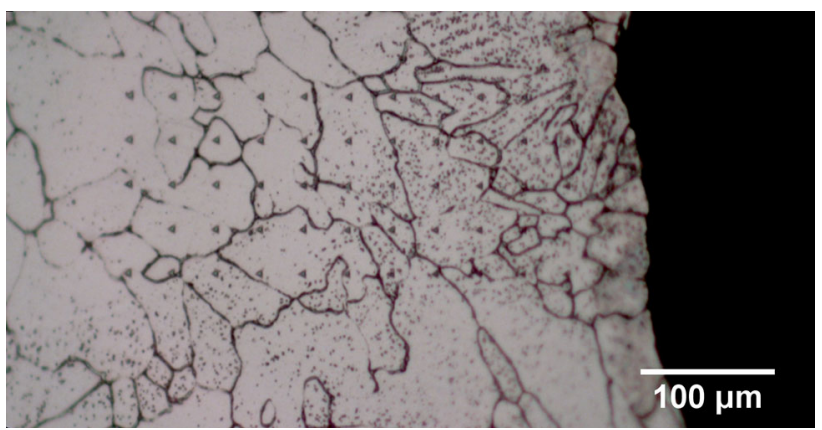

Fig. 13 Detail of indentations performed in the "Light"-density lattice (see Fig. 12)

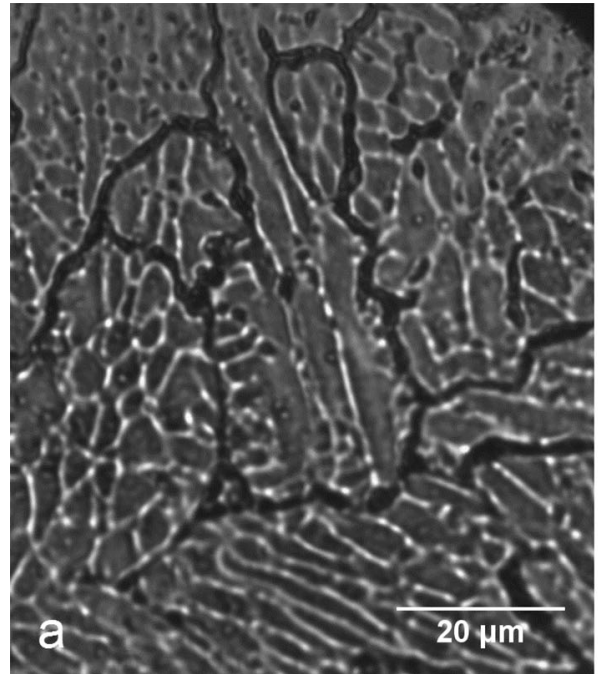

of size, although somewhat larger in the lattice structure with an approximated 1-5 $\mu \mathrm{m}$ range compared to $0.5-2 \mu \mathrm{m}$ for the solid sample in these two compared cross sections.

3.3.1 Intrinsic 316LN Hardness. The variation in $\mathrm{H}$ as a function of $\mathrm{h}$ for all indentations performed in the lattices with Light, Medium and Heavy density, as well as for the solid sample indented as a reference, is presented in the average curves in Fig. 15(a).

A similar behavior in the hardness curves can be observed for all samples indented. At low penetration depths, $316 \mathrm{LN}$ material both in solid and in lattice configurations exhibits high $H$; a value of around $6 \mathrm{GPa}$ can be read at $100 \mathrm{~nm}$ depth $\left(\mathrm{H}_{100}\right)$. Hardness decreases uniformly as the indenter penetrates deeper into the material, down to values of around $4 \mathrm{GPa}$ measured at the maximum penetration depth $(h=500)$. Individual values of hardness for the different specimens at characteristic indentation depths are presented in Table 5.

The increase in the hardness (and the reduction in plasticity) with the decrease in the penetration depth is a well-known phenomenon regarded as "indentation size effect-ISE," which becomes more important at depths of less than approximately $1 \mu \mathrm{m}$ (Ref 29). The interaction between dislocations and the microstructural features of the material, such as the grain boundaries, plays a major role (Ref 30, 31). ISE phenomenon has been widely reported for different metallic materials and alloys, including austenitic stainless steels such as 304L, 316L (Ref 31, 32). In the case of this research, and accounting for the penetration depth regime of the indentations performed $\left(h_{\max }=0.5 \mu \mathrm{m}\right)$, and the length scale of the subgrain structure of the $316 \mathrm{LN}$ material evaluated $\left(d_{\text {sub }} \approx 2 \mu \mathrm{m}\right.$, see Fig. 14), it may be stated that the decrease observed in the hardness with the penetration depth is associated with an indentation size effect. As mentioned before, sub-grains in additively manufactured $316 \mathrm{LN}$ are formed due to segregation of alloying elements, which is always accompanied by accumulation of dislocations in the sub-grain boundaries. Previous investigations of the hardness by conventional methods and micro-hardness of laser-processed 316L, with comparable microstructures, indicate that such dislocations have both a hardening and strengthening effect in the material,

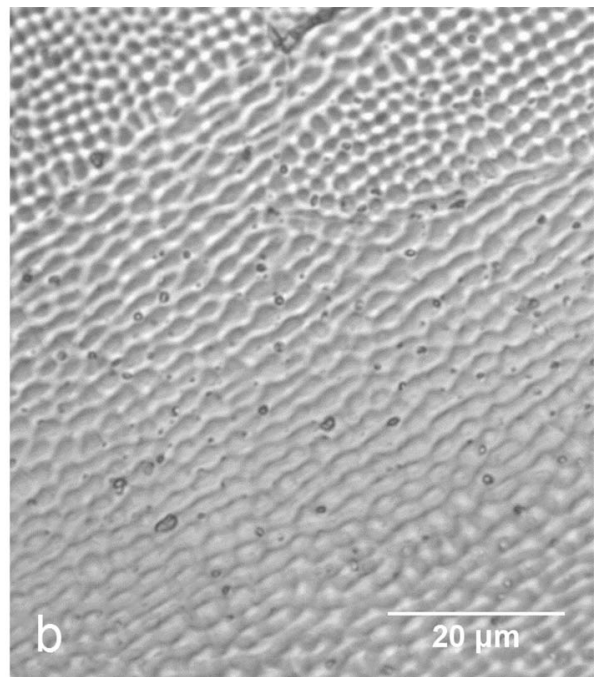

Fig. 14 Sub-grain microstructure comparison of a lattice structure (a) and a solid (b) 316LN sample. Approximately 1-5 $\mu \mathrm{m}$ sub-grain size range for the lattice structure compared to $0.5-2 \mu \mathrm{m}$ for the solid sample 

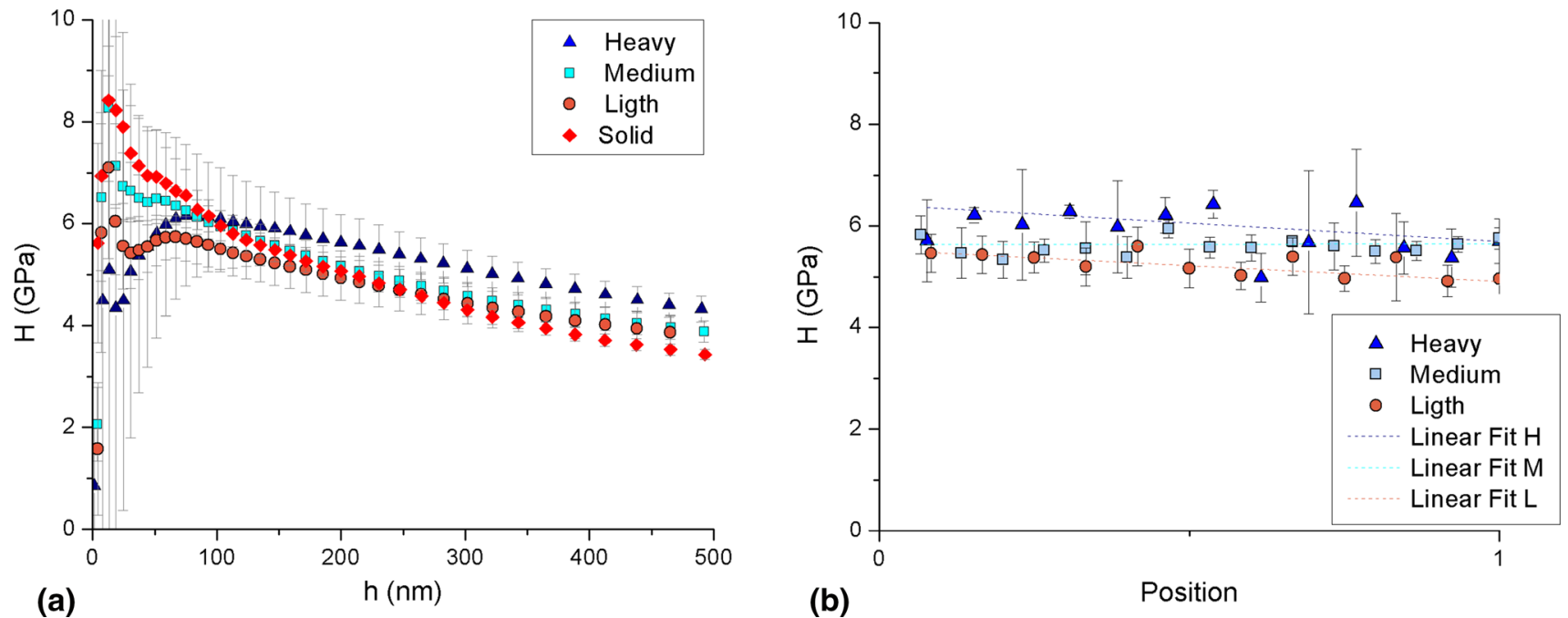

Fig. 15 Average curves of $H$ vs. $h$ for indentations performed in the solid and in the Light, Medium and Heavy lattices (a). Profile with the variation in the hardness $\left(H_{100-200}\right)$ across the cross section of the lattices' struts $(b)$

Table 5 Average characteristic hardness values as measured at 100 and $500 \mathrm{~nm}$ penetration depth

\begin{tabular}{lccrr}
\hline & & 316LN Lattice & & \\
\cline { 2 - 4 } & \multicolumn{1}{c}{ Light } & Medium & Heavy & Solid 316LN \\
\hline$H_{100}, \mathrm{GPa}$ & $5.5 \pm 0.5$ & $5.9 \pm 0.3$ & $6.1 \pm 1.0$ & $6.0 \pm 0.3$ \\
$H_{500}, \mathrm{GPa}$ & $3.9 \pm 0.3$ & $3.9 \pm 0.2$ & $4.3 \pm 0.2$ & $3.4 \pm 0.1$ \\
$E_{100}, \mathrm{GPa}$ & $185.2 \pm 18.9$ & $183.0 \pm 9.3$ & $156.4 \pm 22.5$ & $190.2 \pm 11.7$ \\
$E_{500}, \mathrm{GPa}$ & $136.2 \pm 25.3$ & $126.0 \pm 9.2$ & $97.5 \pm 20.7$ & $173.4 \pm 3.5$ \\
\hline
\end{tabular}

due to a pinning effect (Ref 13, 33, 34). It may then be speculated that the size effect observed in the EBM $316 \mathrm{LN}$ materials evaluated is related to the interaction between the dislocations induced and activated by the indentation and those at the sub-grain boundaries.

It deserves to be highlighted that the hardness of the different evaluated lattices was comparable and even slightly higher than that of the solid material. That can be evidenced when comparing the values obtained at the maximum penetration depth $h_{\max }=500 \mathrm{~nm}$ (see Table 5). The differences in the size and features of the sub-grain structure between the solid and lattice, the process parameters as well as the solidification conditions are quite different, which might have an effect on the differences observed in the intrinsic hardness.

In Fig. 15(b), the hardness measured in the lattice specimens with different densities is presented as a function of the thickness of the struts, from the position 0 (center of the strut) to 1 (periphery), as illustrated in Fig. 13. In this case, hardness values $\left(H_{100-200}\right)$ are averaged in the depth range $h=100$ $200 \mathrm{~nm}$. Dispersion of the results at different levels of the struts' cross sections is probably ascribed to the complexity of the grain and sub-grain microstructures, in which within one single grain, several sub-grains with different sizes and orientations are shown in Fig. 14). It is interesting that, despite the aforementioned dispersion of results, uniform hardness values are observed across the cross section, which indicates mechanical and structural consistency of the material in the lattices.
3.3.2 Intrinsic 316LN Elastic Modulus. The variation in $E$ as a function of $h$ is presented in the average curves in Fig. 16(a). In the case of the solid 316LN sample, a relatively constant behavior in the elastic modulus is observed for all the penetration regimes. In the case of the lattices, especially the Light and Medium densities, an elastic modulus value comparable to that of solid material can be read from the graphs at lower penetration depths $(h=100 \mathrm{~nm})$. A somewhat lower value is observed for the lattice with the Heavy density. Furthermore, a continuous decrease in $\mathrm{E}$ is evidenced as the indenter further penetrates the material. In this case, it becomes necessary in the analysis to account for the loading scenario for nanoindentation when performed in lattice configurations. Although the tests are conducted perpendicular to the cross section of the struts, the stiffness of the system is affected principally by two factors: (1) the geometric configuration of the lattices, since the beams are angled with respect to the indentation axis allowing for bending to occur and (2) specimens are embedded polymeric resin, with a much lower stiffness than the indented $316 \mathrm{LN}$ material. At the lower penetration regime $(50-100 \mathrm{~nm})$, the effect of the external factors mentioned in the measurements is minor, and extracted $E$ values approximate the intrinsic $316 \mathrm{LN}$ properties. The effect of external factors plays an increasing role in the apparent $E$ measured as the indenter progresses toward the maximum penetration depth. Despite the lower values exhibited by the lattice with Heavy density, the intrinsic stiffness measured in the lattices $E_{100} \approx 156$ to $185 \mathrm{GPa}$ is comparable with that of 

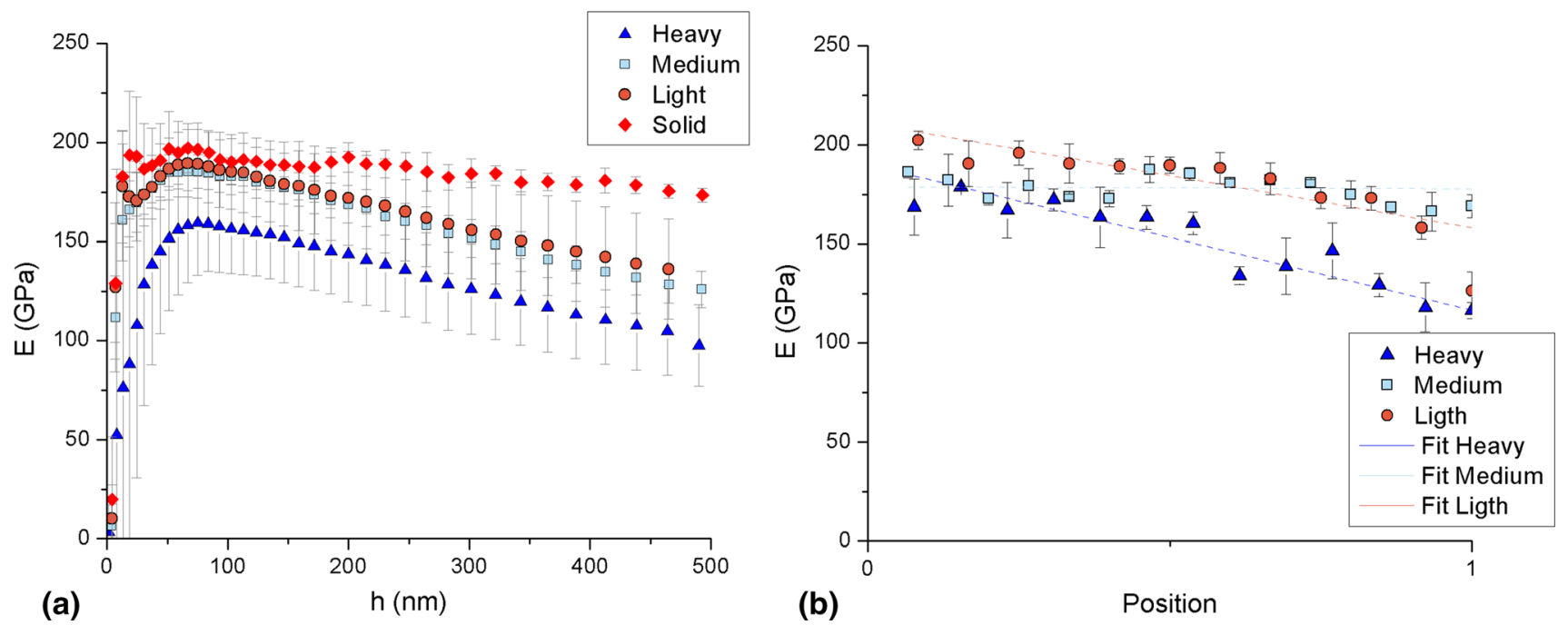

Fig. 16 Average curves of $E$ vs. $h$ for indentations performed in the solid and in the Light, Medium and Heavy lattices (a). Profile with the variation in the elastic modulus $\left(E_{100-200}\right)$ across the cross section of the lattices' struts (b)

the solid $E_{100} \approx 190 \mathrm{GPa}$ and with the elastic modulus values reported in the literature for $316 \mathrm{~L}$ stainless steel measured by nanoindentation tests (Ref 35$)$.

A slight drop in the elastic modulus values is also observed as the indentations approach the periphery of the struts' cross section. This trend is more accentuated in the lattice with Heavy density. It is then possible that the experimental factors discussed previously have also an increasing impact in the measurements as they approach the periphery of the lattice struts. Nevertheless, as for the hardness, it can be confirmed that the locally assessed mechanical behavior of the $316 \mathrm{LN}$ is uniform throughout the cross section of the lattices.

\section{Conclusions}

1. Electron beam melting technology can successfully be used to fabricate lightweight lattice structures in $316 \mathrm{LN}$ stainless steel.

2. Compression behavior of the EBM-manufactured lattices with octagon cells from $316 \mathrm{~L}$ austenitic stainless steel and from Ti6Al4V is substantially different. Stainless steel lattices exhibit elastic and further-plastic deformation until almost complete collapse of the structure, while Ti6Al4V ones do not show much plasticity and often exhibit brittle type collapse.

3. Compressive strength of the fabricated $316 \mathrm{~L}$ lattice structures was in the range of $0.6-5 \mathrm{MPa}$ depending on EBM process settings. It was possible to modify the lattice structure porosity and resulting compression strength by changing manufacturing process parameters alone without altering the design CAD files.

4. Grain size distribution of the lattice structures is wide but significantly finer than that of solid material manufactured using EBM. Sub-grain structure is in the same size range, but the diameter varies between 1 and $5 \mu \mathrm{m}$ for the lattices and $0.5-2 \mu \mathrm{m}$ for the solid sample in the cross sections examined.
5. Intrinsic mechanical behavior in terms of hardness and elastic modulus of the $316 \mathrm{LN}$ in the studied lattices was locally assessed using nanoindentation. Measured properties are comparable with those found on the solid 316LN counterparts. Locally evaluated mechanical properties are uniform across the thickness of the individual struts in the different lattices studied.

6. Grain boundary precipitates show increased levels of chromium, molybdenum and silicon.

\section{Acknowledgments}

Open access funding provided by Mid Sweden University. Authors acknowledge Dr. E. Jiménez-Piqué and personnel at CIEFMA (UPC) for their assistance in nanoindentation experiments. Research is conducted with financial support from the European Regional Development Fund through the Interreg Sweden-Norway program.

\section{Open Access}

This article is distributed under the terms of the Creative Commons Attribution 4.0 International License (http://creativecommons.org/ licenses/by/4.0/), which permits unrestricted use, distribution, and reproduction in any medium, provided you give appropriate credit to the original author(s) and the source, provide a link to the Creative Commons license, and indicate if changes were made.

\section{Author Contributions}

Fabrication and microstructural analysis were performed by Stefan Roos. Nanoindentation and hardness analyses were performed by Dr. Carlos Botero. Mechanical compression testing of lattices was performed by Stefan Roos and Dr. Jonas Danvind. 


\section{References}

1. W.E. Frazier, Metal Additive Manufacturing: A Review, J. Mater. Eng. Perform., 2014, 23(6), p 1917-1928

2. S.L. Sing, J. An, W.Y. Yeong, and F.E. Wiria, Laser and Electron-Beam Powder-Bed Additive Manufacturing of Metallic Implants: A Review on Processes, Materials and Designs, J. Orthop. Res., 2016, 34(3), p 369-385

3. J. Karlsson, in Optimization of Electron Beam Melting for Production of Small Components in Biocompatible Titanium Grades, Doctoral thesis, comprehensive summary, Acta Universitatis Upsaliensis, Uppsala, 2015

4. P. Hallberg, Application Development manager at Arcam AB, Personal communication via E-mail 'Offset, funktion och resultat', 03 March 2017

5. X.Y. Cheng et al., Compression Deformation Behavior of Ti-6Al-4V Alloy with Cellular Structures Fabricated by Electron Beam Melting, $J$. Mech. Behav. Biomed. Mater, 2012, 16, p 153-162

6. C. Neff, N. Hopkinson, N.B. Crane, Selective Laser Sintering of Diamond Lattice Structures: Experimental Results and FEA Model Comparison, in Presented at the Solid Freeform Fabrication, Texas, 2015, pp. 1104-1117

7. C. de Formanoir, M. Suard, R. Dendievel, G. Martin, and S. Godet, Improving the Mechanical Efficiency of Electron Beam Melted Titanium Lattice Structures by Chemical Etching, Addit. Manuf., 2016, 11, p 71-76

8. L. Xiao, W. Song, C. Wang, H. Liu, H. Tang, and J. Wang, Mechanical Behavior of Open-Cell Rhombic Dodecahedron Ti-6Al-4V Lattice Structure, Mater. Sci. Eng. A, 2015, 640, p 375-384

9. L. Xiao et al., Mechanical Properties of Open-Cell Rhombic Dodecahedron Titanium Alloy Lattice Structure Manufactured Using Electron Beam Melting under Dynamic Loading, Int. J. Impact Eng., 2017, 100, p 75-89

10. S.Y. Choy, C.-N. Sun, K.F. Leong, and J. Wei, Compressive Properties of Ti-6Al-4V Lattice Structures Fabricated by Selective Laser Melting: Design, Orientation and Density, Addit. Manuf., 2017, 16, p 213-224

11. V. Crupi, E. Kara, G. Epasto, E. Guglielmino, and H. Aykul, Static Behavior of Lattice Structures Produced Via Direct Metal Laser Sintering Technology, Mater. Des., 2017, 135, p 246-256

12. Q. Feng et al., Quasi-Static Analysis of Mechanical Properties of Ti6A14V Lattice Structures Manufactured Using Selective Laser Melting, Int. J. Adv. Manuf. Technol., 2018, 94(5), p 2301-2313

13. Y.M. Wang et al., Additively Manufactured Hierarchical Stainless Steels with High Strength and Ductility, Nat. Mater., 2017, 17, p 63

14. Z. Xiao, Y. Yang, R. Xiao, Y. Bai, C. Song, and D. Wang, Evaluation of Topology-Optimized Lattice Structures Manufactured Via Selective Laser Melting, Mater. Des., 2018, 143, p 27-37

15. M. Fousová, J. Kubásek, D. Vojtěch, J. Fojt, and J. Čapek, 3D Printed Porous Stainless Steel for Potential Use in Medicine, IOP Conf. Ser. Mater. Sci. Eng., 2017, 179(1), p 012025

16. Y. Zhong et al., Additive Manufacturing of $316 \mathrm{~L}$ Stainless Steel by Electron Beam Melting for Nuclear Fusion Applications, J. Nucl. Mater., 2017, 486, p 234-245

17. L.-E. Rännar, A. Koptyug, J. Olsén, K. Saeidi, and Z. Shen, Hierarchical Structures of Stainless Steel 316L Manufactured by Electron Beam Melting, Addit. Manuf., 2017, 17(Supplement C), p $106-112$

18. X. Li and B. Bhushan, A Review of Nanoindentation Continuous Stiffness Measurement Technique and Its Applications, Mater. Charact., 2002, 48(1), p 11-36
19. E. Broitman, Indentation Hardness Measurements at Macro-, Micro-, and Nanoscale: A Critical Overview, Tribol. Lett., 2016, 65(1), p 23

20. ISO 13314:2011(E), Mechanical Testing of Metals-Ductility Testing-Compression Test for Porous and Cellular Metals

21. W.C. Oliver and G.M. Pharr, Measurement of Hardness and Elastic Modulus by Instrumented Indentation: Advances in Understanding and Refinements to Methodology, J. Mater. Res., 2004, 19(1), p 3-20

22. J. Olsén, Z. Shen, L. Liu, A. Koptyug, and L.-E. Rännar, Micro- and Macro-structural Heterogeneities in 316L Stainless Steel Prepared by Electron-Beam Melting, Mater. Charact., 2018, 141, p 1-7

23. D. Wang, C. Song, Y. Yang, and Y. Bai, Investigation of Crystal Growth Mechanism during Selective Laser Melting and Mechanical Property Characterization of 316L Stainless Steel Parts, Mater. Des., 2016, 100, p 291-299

24. M. Ma, Z. Wang, and X. Zeng, A Comparison on Metallurgical Behaviors of 316L Stainless Steel by Selective Laser Melting and Laser Cladding Deposition, Mater. Sci. Eng. A, 2017, 685, p 265-273

25. S. Katayama and A. Matsunawa, Solidification Microstructure of Laser Welded Stainless Steels, Int. Congr. Appl. Lasers Electro-Opt., 1984, 1984(2), p 60-67

26. Z. Sun, X. Tan, S.B. Tor, and W.Y. Yeong, Selective Laser Melting of Stainless Steel 316L with Low Porosity and High Build Rates, Mater. Des., 2016, 104, p 197-204

27. C. Qiu, M.A. Kindi, A.S. Aladawi, and I.A. Hatmi, A Comprehensive Study on Microstructure and Tensile Behaviour of a Selectively Laser Melted Stainless Steel, Sci. Rep., 2018, 8(1), p 7785

28. Y. Zhong et al., Additive Manufacturing of ITER First Wall Panel Parts by Two Approaches: Selective Laser Melting and Electron Beam Melting, Fusion Eng. Des., 2017, 116(Supplement C), p 24-33

29. G.M. Pharr, E.G. Herbert, and Y. Gao, The Indentation Size Effect: A Critical Examination of Experimental Observations and Mechanistic Interpretations, Аnпи. Rev. Mater. Res., 2010, 40(1), p 271-292

30. Z.G. Voyiadjis and M. Yaghoobi, Review of Nanoindentation Size Effect: Experiments and Atomistic Simulation, Crystals, 2017, 7(10), p 102-129

31. F. Pöhl, S. Huth, and W. Theisen, Detection of the Indentation-SizeEffect (ISE) and Surface Hardening by Analysis of the Loading Curvature C, Int. J. Solids Struct., 2016, 84, p 160-166

32. F.K. Yan, B.B. Zhang, H.T. Wang, N.R. Tao, and K. Lu, Nanoindentation Characterization of Nano-twinned Grains in an Austenitic Stainless Steel, Scr. Mater, 2016, 112, p 19-22

33. Y. Zhong, L. Liu, S. Wikman, D. Cui, and Z. Shen, Intragranular Cellular Segregation Network Structure Strengthening 316L Stainless Steel Prepared by Selective Laser Melting, J. Nucl. Mater., 2016, 470, p 170-178

34. K. Saeidi, X. Gao, Y. Zhong, and Z.J. Shen, Hardened Austenite Steel with Columnar Sub-grain Structure Formed by Laser Melting, Mater. Sci. Eng. A, 2015, 625, p 221-229

35. J. Ballarre, E. Jimenez-Pique, M. Anglada, S.A. Pellice, and A.L. Cavalieri, Mechanical Characterization of Nano-reinforced Silica Based Sol-Gel Hybrid Coatings on AISI, 316L Stainless Steel Using Nanoindentation Techniques, Surf. Coat. Technol., 2009, 203(20), p $3325-3331$

Publisher's Note Springer Nature remains neutral with regard to jurisdictional claims in published maps and institutional affiliations. 